\title{
In vivo histopathological assessment of the muscularis propria in achalasia by using endocytoscopy (with video)
}

Authors

Institutions
Hiroki Sato ${ }^{1}$, Haruhiro Inoue ${ }^{1}$, Haruo Ikeda' ${ }^{1}$ Chiaki Sato ${ }^{1}$, Esperanza Grace R. Santi ${ }^{2}$, Chainarong Phalanusitthepha', Yutaka Aoyagi ${ }^{3}$, Shin-ei Kudo ${ }^{1}$

${ }^{1}$ Digestive Disease Center, Showa University, Northern Yokohama Hospital, Japan

${ }^{2}$ Department of Gastroenterology, De La Salle University Medical Center, Philippines

${ }^{3}$ Division of Gastroenterology, Niigata University Medical and Dental Hospital, Japan submitted 23. April 2014 accepted after revision 5. May 2014

\section{Bibliography}

Dol http://dx.doi.org/

10.1055/s-0034-1377319

Published online: 0.0 .

Endoscopy International Open

2014; 2: E178-E182

(c) Georg Thieme Verlag KG

Stuttgart · New York

E-ISSN 2196-9736

\section{Corresponding author}

\section{Hiroki Sato, MD}

Showa University Northern

Yokohama Hospital - Digestive Disease Center

Chigasaki-chuo 35-1

Yokohama 224-8503

Japan

Fax: +81-45-949-7927

pyloki@yahoo.co.jp
Background: The histopathology of the muscularis propria (MP) is unknown in patients with achalasia. Endocytoscopy (EC) was developed as an ultra-high magnification endoscopy, and the submucosal tunnel created during peroral endoscopic myotomy (POEM) not only provides access to the MP but also enables subsequent endoscopic assessment of the MP.

Patients and methods: In seven patients with achalasia (mean \pm SD; $35 \pm 18.1$ years; men:women, 4:3) who underwent POEM (myotomy length: $12 \pm 2.2 \mathrm{~cm}$ ), subsequent EC examination was performed from the mid-esophagus to the gastric side. EC images were compared to the results of histopathologic examination (two biopsies from

\section{Introduction \\ $\nabla$}

Ultra-high magnification endoscopy, also known as endocytoscopy (EC) or endomicroscopy, enables assessment of the muscularis propria (MP) or myenteric plexus in vivo through a submucosal tunnel [ $1-3]$. This technique is expected to be applicable for clinical use for the treatment of neuromuscular diseases because the nidus of such diseases is under the mucosa, a location that is difficult to assess using conventional endoscopy. Achalasia is an idiopathic esophageal motility disorder characterized by a lack of peristalsis in the esophageal body and absent or incomplete relaxation of the lower esophageal sphincter (LES) [45]. The novel procedure, peroral endoscopic myotomy (POEM), has become one of the best treatment options for achalasia because it is safe, offers long-lasting symptom control, and is less invasive than surgery [6-8]. Moreover, the submucosal tunnel created during POEM allows insertion of the endoscope under the mucosa and access to the MP. During POEM, thick muscle at the middle to lower esophagus, as well as thin muscle at the the mid-esophagus and lower esophageal sphincter), which was the standard.

Results: In all patients, favorable EC images were obtained, and spindle-shaped smooth muscle cells were detected. In our series, we observed no notable features such as atrophy or hypertrophy of smooth muscle cells. In addition, the EC assessment was consistent with the results of biopsy. No complications were encountered during any of the procedures.

Conclusion: In a clinical setting, real-time assessment of the MP using EC is feasible. This technique may play an important role in determining the pathology of achalasia and other diseases that affect gastrointestinal function.

there are variations among patients with achalasia $[9,10]$.

The histopathology of achalasia has been insufficiently investigated because of the low prevalence of this condition, and because the mainstream treatment for achalasia has been balloon dilatation, which does not allow tissue sampling. Surgical myotomy may allow access to the MP at the LES but, because of the limited treatment window (difficult to pull the mid-esophagus into the abdominal cavity), using surgical myotomy to assess the mid-esophagus is technically difficult. The approach to and examination of the esophageal MP was difficult until the introduction of POEM.

The purpose of the current study, therefore, is to determine the feasibility of EC for real-time histopathologic examination of the MP in a clinical setting, and to provide more knowledge about the histopathologic features of esophageal tissues features of esophageal tissues in achalasia.

\section{Patients and Methods \\ Patients}

This study was performed at Showa University Northern Yokohama Hospital, a tertiary referral center in Japan. Enrollment began in December 

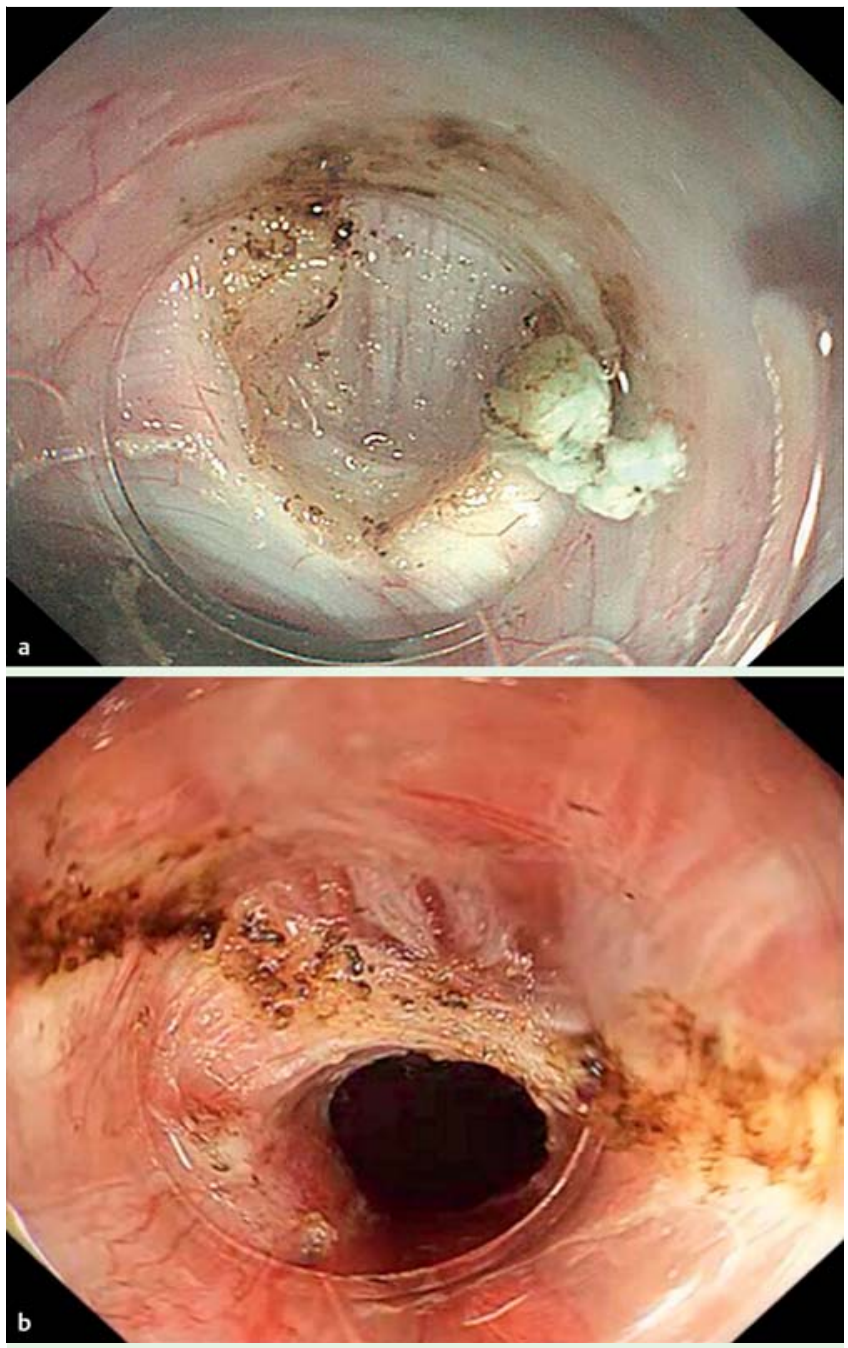

Fig. 1 During peroral endoscopic myotomy, thick muscle is observed at the mid- to lower- esophagus (a), whereas thin muscle is observed at the LES (b).

2013 and patients with consecutive achalasia who were candidates for POEM were recruited for this study. Patients younger than 18 years and those whose health was not favorable for enrollment because of severe comorbidity in organs such as the heart or lungs were excluded from this study. In instances where full-thickness myotomy was performed, EC examination was not performed, and these patients were also excluded from the analysis. The current study was approved by the Institutional Review Board (No.1311-05). Written informed consent was obtained from all participants, and the study was conducted according to the Declaration of Helsinki.

\section{Peroral endoscopic myotomy}

POEM was performed following the technique described by Inoue [6]. Before the procedure, esophageal contents were cleared by endoscopic suction using a $3.7-\mathrm{mm}$ channel endoscope (GIF1T240; Olympus Co., Tokyo, Japan) under intravenous anesthesia to prevent aspiration caused by intubation. Under general anesthesia with positive pressure ventilation, the endoscopic procedure began with $\mathrm{CO}_{2}$ insufflation. After submucosal injection at the level of the mid- esophagus, a 2-cm longitudinal mucosal incision was created as the point of entry. A one-third circumferential submucosal tunnel was created from entry down to the LES

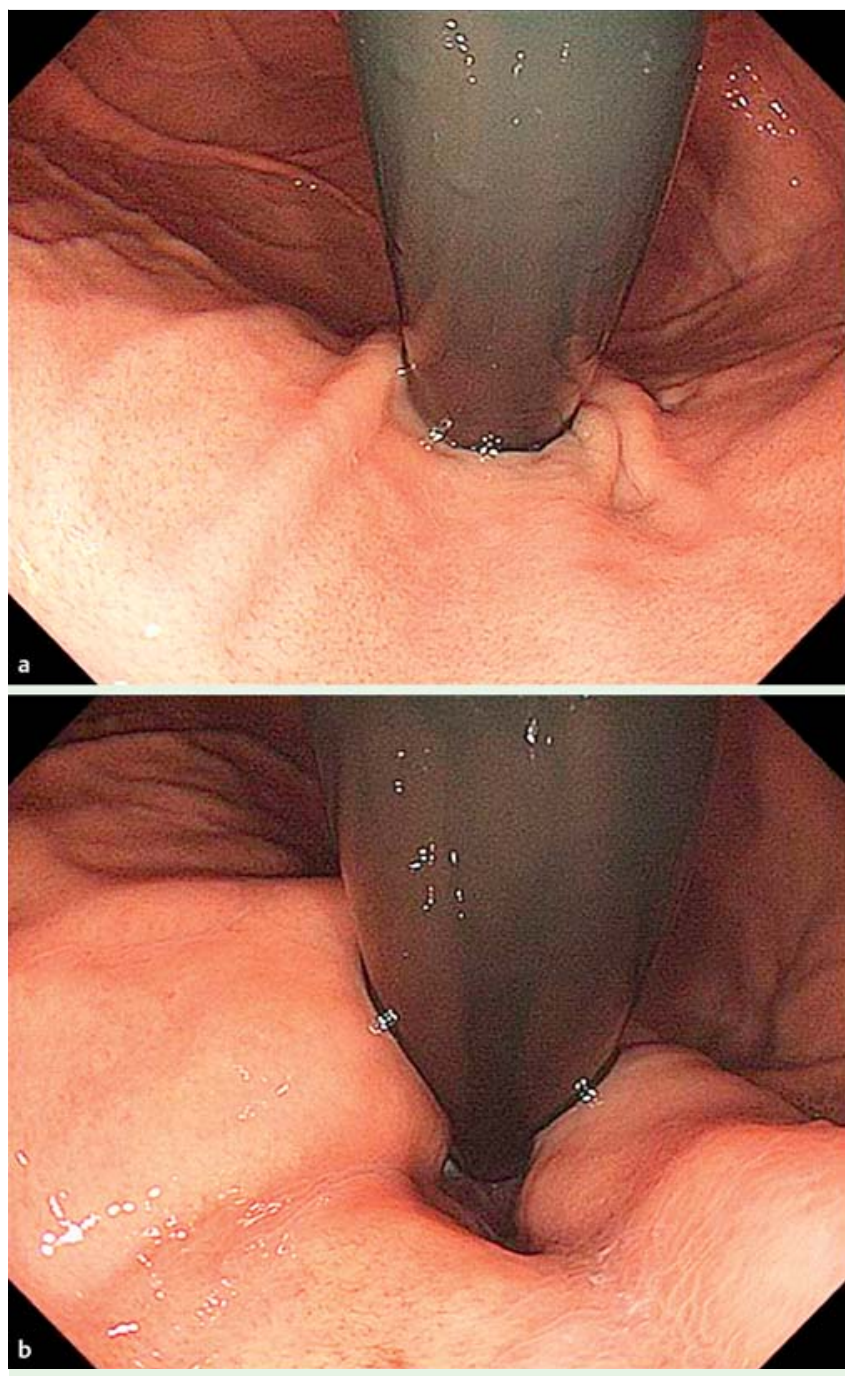

Fig. 2 As compared to pre-POEM (a), a totally relaxed esophagogastric junction is seen on retroflex view from the gastric side with POEM (b).

and into approximately $2-3 \mathrm{~cm}$ of the gastric side. Dissection of the circular muscle (from $2 \mathrm{~cm}$ distal to the mucosal entry) was performed at the center of the submucosal tunnel. After myotomy, a totally relaxed C (EGJ) was confirmed by inserting an endoscope into the natural lumen down to the gastric side, and by a retroflex view from the gastric side to EGJ ( $\bullet$ Fig. 2). The mucosal entry was closed with hemostatic clips.

\section{Endocytoscopy}

All EC examinations were performed using the integrated-type endocytoscope GIF-Y0002 (prototypes from Olympus Co.). GIFY0002 has one lens that can consecutively increase the magnification from the conventional endoscopy level to $380 \times$ magnification (tissue field of view, $700 \times 600 \mu \mathrm{m}$ ) using a hand lever. The GIF-Y0002 allows gradual magnification at the center of the monitor, thus ensuring accurate localization of the area being viewed. For EC, a mixture of $0.1 \%$ methylene blue was used to stain the tissues and to obtain images similar to those obtained in conventional pathology examination. Methylene blue staining reveals details of cell structure, including nuclei and cytoplasm. 
Table 1 Characteristics of patients with achalasia.

\begin{tabular}{|c|c|c|c|c|c|c|c|}
\hline Case & Age & Gender & $\begin{array}{l}\text { Duration of symptom } \\
\text { (years) }\end{array}$ & Manometry & $\begin{array}{l}\text { Myotomy length } \\
(\mathrm{cm})\end{array}$ & $\begin{array}{l}\text { EC } \\
\text { diagnosis }\end{array}$ & $\begin{array}{l}\text { Histopathologic } \\
\text { diagnosis }\end{array}$ \\
\hline 1 & 78 & M & 7 & - & 14 & Normal & n.p. \\
\hline 2 & 71 & M & 20 & Type II & 9 & Normal & n.p. \\
\hline 3 & 51 & M & 4 & Type I & 12 & Normal & n.p. \\
\hline 4 & 57 & $\mathrm{~F}$ & 20 & Type II & 12 & Normal & n.p. \\
\hline 5 & 19 & $\mathrm{~F}$ & 3 & Type II & 12 & Normal & n.p. \\
\hline 6 & 33 & M & 4 & Type II & 16 & Normal & n.p. \\
\hline 7 & 29 & $\mathrm{~F}$ & 7 & - & 11 & Normal & n.p. \\
\hline
\end{tabular}

M: male, F: female, n.p.: nothing particular

Manometry: manometric findings according to Chicago classification criteria [13] [14]: manometry was not completed

Endocytoscopy (EC) images showed no evidence of atrophy or hypertrophy, which was consistent with the diagnosis established by histopathology.

\section{Study procedure}

EC examination was performed after POEM and prior to closure of the mucosal entry site. After staining with $2 \mathrm{~mL}$ of methylene blue, the head of the endocytoscope was moved closer to the surface of the MP. The EC examination was started from the proximal $2 \mathrm{~cm}$ of circular muscle through remnant circular muscle into the gastric side. Longitudinal muscle was also examined. Two biopsy specimens were obtained from the proximal circular muscle and at the LES area (high-pressure zone) for histopathological assessment as confirmation of in vivo diagnosis. EC findings were assessed according to the previous in vivo report and histopathology reports $[1,11,12]$. EC findings of the MP were classified as: atrophy, normal, or hypertrophy. Biopsy specimens were assessed by a pathologist blinded to EC images.

\section{Results \\ $\nabla$}

Seven patients with achalasia who underwent POEM were enrolled in this study and underwent concomitant EC examination. The mean $( \pm S D)$ age of the participants was $35.0 \pm 18.1$ years, and the study population consisted of four male and three female patients. The duration of achalasia symptoms was $5.5 \pm 7.5$ years, and ranged from 3 to 20 years. According to Chicago classification criteria $[13,14]$, manometric findings were classified as type I in one patient and type II in four patients; in two patients, manometry was not completed because of difficulty in inserting the catheter through the LES.The total length of the endoscopic myotomy was $12 \pm 2.2 \mathrm{~cm}$ with a mean of $10 \pm 2.0 \mathrm{~cm}$ in the esophagus and $3.0 \pm 0.69 \mathrm{~cm}$ in the stomach ( $\bullet$ Table 1 ).

Favorable EC images were obtained, and spindle-shaped smooth muscle cells were observed. A strong staining nucleus, surrounding cell bodies, and vessels in the muscular layer were visualized. However, none of these structures showed particularly notable changes, that is, non-atrophy and non-hypertrophy of the MP ( $\bullet$ Fig. 3). Moreover, EC findings were consistent with biopsy results ( Fig. 4). In other words, changing patterns such as atrophy or hypertrophy were not identified on the biopsy specimens. EC and biopsy were performed within 10 minutes with no complications. On postoperative day 2 , a liquid diet was started, and on day 4 , all the patients were discharged on a normal diet.

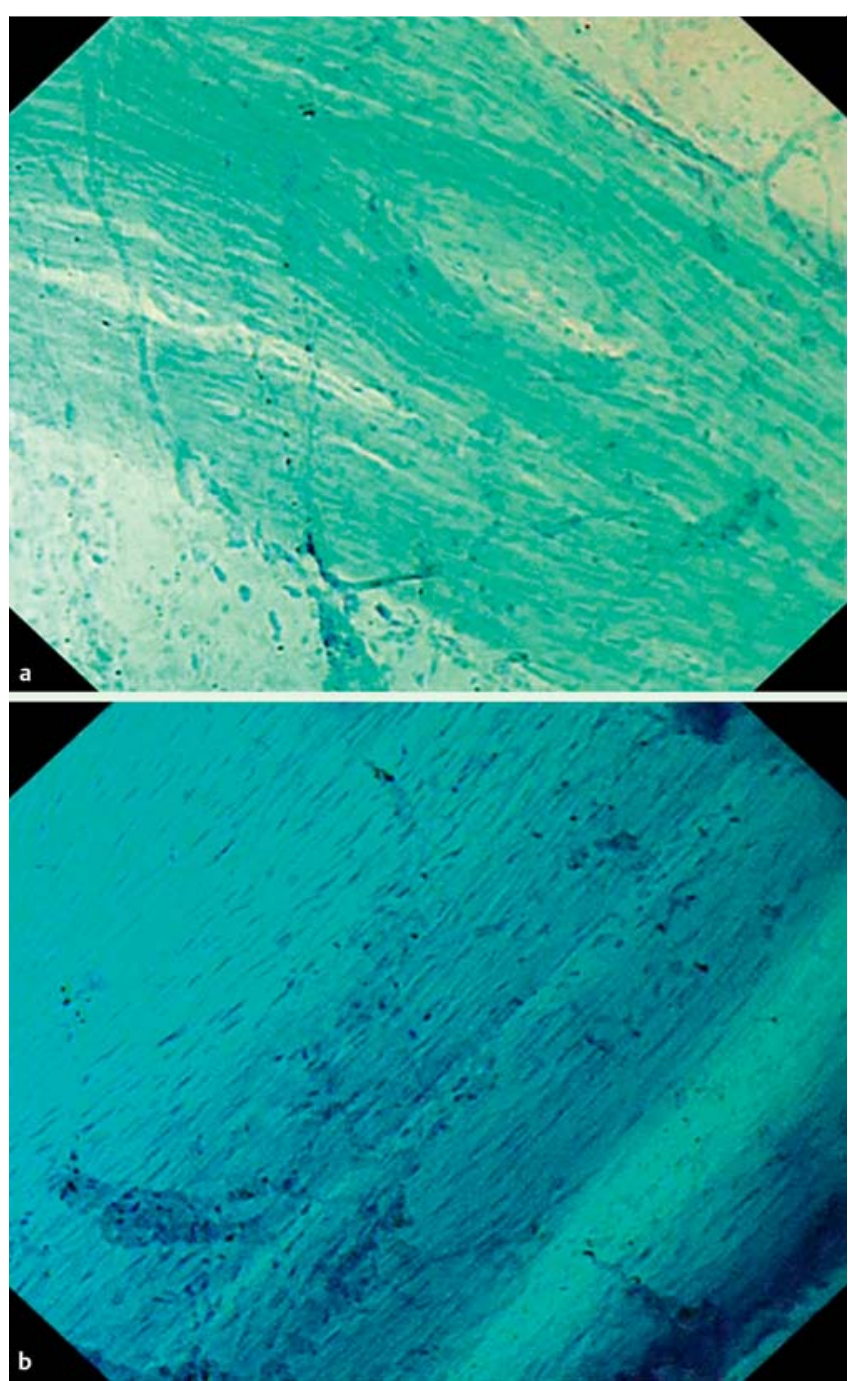

Fig. 3 In vivo images of the muscularis propria of circular and longitudinal muscle in achalasia obtained by endocytoscopy. Spindle-shaped smooth muscle cells, including the nucleus and cell bodies, are clearly identified.

\section{Discussion \\ $\nabla$}

To our knowledge, this is the first study to report in vivo histopathology of the MP in patients with achalasia. Our results offer the following propositions. First, EC can be used for in vivo real-time assessment not only for intestinal mucosa but also for MP via a submucosal tunnel created after POEM. In POEM for achalasia, 


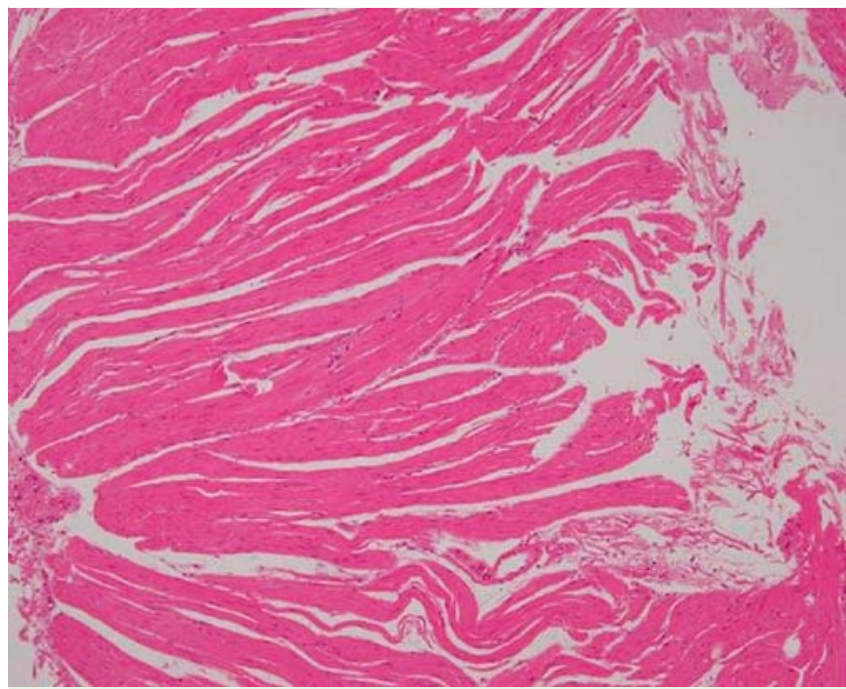

Fig. 4 Conventional microscopic analysis of the muscularis propria shows the smooth muscle layer from one of the achalasia patients with well-preserved spindle-shaped, smooth muscle cells.

real-time histopathological assessment of MP may be helpful to identify the morbid lesion preoperatively, although no atrophy or hypertrophy was identified in our small cohort. The intermittent and time-consuming biopsy method, which also includes adverse event risks such as bleeding, cannot substitute for the continuous and real-time EC assessment. Furthermore, if EC can be performed by resecting the mucosal/submucosal layer using endoscopic mucosal resection/endoscopic submucosal dissection, EC is potentially effective for the diagnosis of other disorders with a nidus located in the MP.

Second, the endocytoscopic morphology of the MP showed no evidence of hypertrophy or atrophy of smooth muscle cells despite a thick or thin appearance on a conventional endoscopic view, and the EC findings were confirmed by histology. This result suggests that the thick or thin appearance of MP in achalasia is caused by the changing histopathology rather than hypertrophy or atrophy, or that such change requires long-term symptoms. Previous studies have examined muscle biopsies from patients with achalasia treated at an earlier stage of disease. These studies have detected intact MP in these patients who had a shorter duration of symptoms and a non-dilated esophagus [15-17], findings that are almost consistent with our results.

This study has several limitations. First, the staining technique used in this series allowed the evaluation of cell structure, cytoplasm, and nuclei alone. The nerve plexuses were not visualized in this setting because of a lack of neuron-specific fluorescent stain available for safe use in humans. Development of stains and methods to evaluate stain toxicity and long-term effects are essential. In the current study, the nerve plexus was not identified by biopsy, probably because the tissue sampling should be nonconsecutive, and with a small specimen. It is expected that EC will help identify the nerve plexus in clinical settings.

\section{Video 1}

online content including video sequences viewable at: www.thieme-connect.de
In addition, achalasia and the other esophageal motility disorders have multidimensional etiology and pathogenesis. Therefore, the associated histopathologic changes are thought to be diverse. The most familiar histomorphologic change is a loss of ganglion cells in the Auerbach plexus of smooth muscle; however, controversy exists regarding the trigger, extent, and degree of the change $[10,18,19]$. Further studies involving a larger series, particularly including participants with a longer duration of symptoms, is warranted. However, the application of this technique should remain limited and performed only in select centers so that POEM and EC can be performed safely.

In conclusion, in vivo real-time assessment of the MP is possible using EC. This technique may play an important role in determining the pathology of achalasia. ( Video $\mathbf{1}$ ).

\section{Abbreviations}

$\nabla$

$\begin{array}{ll}\text { EGJ } & \text { esophagogastric junction } \\ \text { LES } & \text { Lower esophageal sphincter } \\ \text { MP } & \text { Muscularis propria } \\ \text { POEM } & \text { Peroral endoscopic myotomy }\end{array}$

Competing interests: None.

\section{References}

1 Sumiyama $K$, Tajiri $H$, Kato $F$ et al. Pilot study for in vivo cellular imaging of the muscularis propria and ex vivo molecular imaging of myenteric neurons (with video). Gastrointest Endosc 2009; 69: 1129-1134

2 Sumiyama K, Kiesslich R, Ohya TR et al. In vivo imaging of enteric neuronal networks in humans using confocal laser endomicroscopy. Gastroenterology 2012; 143: 1152 - 1153

3 Ohya TR, Sumiyama K, Takahashi-Fujigasaki J et al. In vivo histologic imaging of the muscularis propria and myenteric neurons with probe-based confocal laser endomicroscopy in porcine models (with videos). Gastrointest Endosc 2012; 75: 405-410

4 Cohen S. Motor disorders of the esophagus. N Engl J Med 1979; 301: $184-192$

5 Park W, Vaezi MF. Etiology and pathogenesis of achalasia: the current understanding. Am J Gastroenterol 2005; 100: 1404-1414

6 Inoue $H$, Minami $H$, Kobayashi $Y$ et al. Peroral endoscopic myotomy (POEM) for esophageal achalasia. Endoscopy 2010; 42: 265-271

7 Onimaru $M$, Inoue $H$, Ikeda $H$ et al. Peroral endoscopic myotomy is a viable option for failed surgical esophagocardiomyotomy instead of redo surgical Heller myotomy: a single center prospective study. Journal of the American College of Surgeons 2013; 217: 598-605

8 Swanstrom LL, Kurian A, Dunst CM et al. Long-term outcomes of an endoscopic myotomy for achalasia: the POEM procedure. Ann Surg 2012; 256: 659-667

9 Pehlivanov N, Liu J, Kassab GS et al. Relationship between esophageal muscle thickness and intraluminal pressure: an ultrasonographic study. American journal of physiology Gastrointestinal and liver physiology 2001; 280: G1093-G1098

10 Mittal RK, Kassab G, Puckett JL et al. Hypertrophy of the muscularis propria of the lower esophageal sphincter and the body of the esophagus in patients with primary motility disorders of the esophagus. Am J Gastroenterol 2003; 98: 1705-1712

11 Misiewicz JJ, Waller SL, Anthony PP et al. Pharmacology and histopathology of the cardiac sphincter in achalasia. Gut 1968; 9: 726-727

12 Goldblum JR, Rice TW, Richter JE. Histopathologic features in esophagomyotomy specimens from patients with achalasia. Gastroenterology 1996; 111: $648-654$

13 Bredenoord AJ, Fox M, Kahrilas PJ et al. Chicago classification criteria of esophageal motility disorders defined in high resolution esophageal pressure topography. Neurogastroenterology and motility: the official journal of the European Gastrointestinal Motility Society 2012; 24 : $57-65$

14 Pandolfino JE, Fox MR, Bredenoord AJ et al. High-resolution manometry in clinical practice: utilizing pressure topography to classify oesopha- 
geal motility abnormalities. Neurogastroenterology and motility: the official journal of the European Gastrointestinal Motility Society 2009; 21: 796-806

15 Cassella RR, Brown AL Jr, Sayre GP et al. Achalasia of the Esophagus: Pathologic and Etiologic Considerations. Ann Surg 1964; 160: 474487

16 Misiewicz JJ, Waller SL, Anthony PP et al. Achalasia of the cardia: pharmacology and histopathology of isolated cardiac sphincteric muscle from patients with and without achalasia. The Quarterly journal of medicine 1969; 38: 17-30
17 Csendes A, Smok G, Braghetto I et al. Histological studies of Auerbach's plexuses of the oesophagus, stomach, jejunum, and colon in patients with achalasia of the oesophagus: correlation with gastric acid secretion, presence of parietal cells and gastric emptying of solids. Gut 1992; 33: 150-154

18 Villanacci V, Annese V, Cuttitta A et al. An immunohistochemical study of the myenteric plexus in idiopathic achalasia. Journal of clinical gastroenterology 2010; 44: 407-410

19 Gockel I, Bohl JR, Doostkam S et al. Spectrum of histopathologic findings in patients with achalasia reflects different etiologies. J Gastroenterol Hepatol 2006; 21: 727-733 